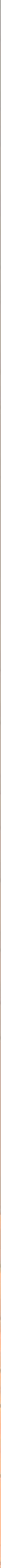




\title{
Cosemisimple Hopf algebras are faithfully flat over Hopf subalgebras
}

\author{
Alexandru Chirvasitu
}

The question of whether or not a Hopf algebra $H$ is faithfully flat over a Hopf subalgebra $A$ has received positive answers in several particular cases: when $H$ (or more generally, just $A$ ) is commutative, cocommutative, or pointed, or when $K$ contains the coradical of $H$. We prove the statement in the title, adding the class of cosemisimple Hopf algebras to those known to be faithfully flat over all Hopf subalgebras. We also show that the third term of the resulting "exact sequence" $A \rightarrow H \rightarrow C$ is always a cosemisimple coalgebra, and that the expectation $H \rightarrow A$ is positive when $H$ is a CQG algebra.

Introduction

1. Preliminaries

2. Main results

3. Expectations on CQG subalgebras are positive

Acknowledgements

References
1179

1182

1187

1192

1197

1197

\section{Introduction}

The issue of faithful flatness of a Hopf algebra (always over a field) over its Hopf subalgebras arises quite naturally in several ways. One direction is via the so-called Kaplansky conjecture [1975], which initially asked whether or not Hopf algebras are free over Hopf subalgebras (as an analogue to the Lagrange theorem for finite groups). The answer was known to be negative, with a counterexample appearing in [Oberst and Schneider 1974], but it is true in certain particular cases: using the notation in the abstract, $H$ is free over $A$ whenever $H$ is finite-dimensional (the Nichols-Zoeller theorem [Montgomery 1993, Theorem 3.1.5]), or pointed [Radford 1977b], or $A$ contains the coradical of $H$ [Radford 1977a, Corollary 2.3].

Montgomery then naturally asks whether one can get a positive result by requiring only faithful flatness of a Hopf algebra over an arbitrary Hopf subalgebra [1993,

MSC2010: primary 16T20; secondary 16T15, 16T05, $20 \mathrm{G} 42$.

Keywords: cosemisimple Hopf algebra, CQG algebra, faithfully flat, right coideal subalgebra, quotient left module coalgebra, expectation. 
Question 3.5.4]. Again, this turns out not to work in general (see [Schauenburg 2000] and also [Chirvasitu 2010], where the same problem is considered in the context of whether or not epimorphisms of Hopf algebras are surjective), but one has positive results in several important cases, such as when $A$ is commutative [Arkhipov and Gaitsgory 2003, Proposition 3.12] or $H$ is cocommutative ([Takeuchi 1972, Theorem 3.2], which also takes care of the case when $H$ is commutative). The most recent version of the question, asked in [Schauenburg 2000], seems to be whether or not a Hopf algebra with bijective antipode is faithfully flat over Hopf subalgebras with bijective antipode.

Another way to get to the faithful flatness issue is via the problem of constructing quotients of affine group schemes. We recall briefly how this goes.

Let $A \rightarrow H$ be an inclusion of commutative Hopf algebras; in scheme language, $A$ and $H$ are affine groups, and the inclusion means that $\operatorname{spec}(A)$ is a quotient group scheme of $\operatorname{spec}(H)$. The Hopf-algebraic analogue of the kernel of this epimorphism is the quotient Hopf algebra $\pi: H \rightarrow C=H / H^{+}$, where $A^{+}$stands for the kernel of the counit of $A$. The map $\pi$ is then normal, in the sense of [Andruskiewitsch and Devoto 1995, Definition 1.1.5]:

$$
\operatorname{LKER}(\pi)=\left\{a \in A \mid(\pi \otimes \mathrm{id}) \circ \Delta(a)=1_{C} \otimes a\right\}
$$

equals its counterpart

$$
\operatorname{RKER}(\pi)=\left\{a \in A \mid(\mathrm{id} \otimes \pi) \circ \Delta(a)=a \otimes 1_{C}\right\} .
$$

This means precisely that $\operatorname{spec}(C)$ is a normal affine subgroup scheme of $\operatorname{spec}(A)$ [Takeuchi 1972, Lemma 5.1]. This gives a map $A \mapsto C$ from quotient affine group schemes of $H$ to normal subgroup schemes. One naturally suspects that this is probably a bijective correspondence, and this is indeed true (see [Takeuchi 1972, Theorem 4.3] and also [Demazure and Gabriel 1970, III §3, 7.2]). In Takeuchi's paper, faithful flatness is crucial in proving half of this result, namely, the injectivity of the map $A \mapsto C$ : one recovers $A$ as $\operatorname{LKER}(\pi)$.

Many of the technical arguments and constructions appearing in this context go through in the noncommutative setting, so one might naturally be led to the faithful flatness issue by trying to mimic the algebraic group theory in a more general setting, where Hopf algebras are viewed as function algebras on a "quantum" group. This is, for example, the point of view taken in the by now very rich and fruitful theory of compact quantum groups, first introduced and studied by Woronowicz: the main characters are certain $C^{*}$ algebras $A$ with a comultiplication $A \rightarrow A \otimes A$ (the minimal $C^{*}$ tensor product), imitating the algebras of continuous functions on compact groups (we refer the reader to [Klimyk and Schmüdgen 1997, Chapter 11] or Woronowicz's landmark papers [1987; 1988] for details). 
These objects are not quite Hopf algebras, but for any compact quantum group $A$ as above, one can introduce a genuine Hopf algebra $\mathscr{A}$, imitating the algebra of representative functions on a compact group (i.e., the linear span of matrix coefficients of finite-dimensional unitary representations), and which contains all the relevant information on the representation theory of the quantum group in question. The abstract properties of such Hopf $(*)$-algebras have been axiomatized, and they are usually referred to as compact quantum group (CQG) algebras (see [Klimyk and Schmüdgen 1997, Section 11.3] or the original paper [Dijkhuizen and Koornwinder 1994], where the term was coined). They are always cosemisimple (as an analogue of Peter-Weyl theory for representations of compact groups), which is why we hope that despite the seemingly restrictive hypothesis of cosemisimplicity, the results in the present paper might be useful apart from any intrinsic interest, at least in dealing with Hopf-algebraic issues arising in the context of compact quantum groups.

We now describe the contents of the paper.

In the first section we introduce the conventions and notation to be used throughout the rest of the paper, and also develop the tools needed to prove the main results. In Section 1A we set up a Galois correspondence between the set of right coideal subalgebras of a Hopf algebra $H$ and the set of quotient left module coalgebras of $H$. We then recall basic results on categories of objects imitating Sweedler's Hopf modules: These have both a module and a comodule structure, one of them over a Hopf algebra $H$, and the other one over a right coideal subalgebra or a quotient left module coalgebra of $H$. These categories are used extensively in the subsequent discussion.

Section 2 is devoted to the main results. We provide sufficient conditions for faithful flatness over Hopf subalgebras in Theorem 2.1 and Corollary 2.4. We also investigate the case of cosemisimple $H$ further, proving in Theorem 2.5 that for any Hopf subalgebra $A$, the quotient left $H$-module coalgebra $C=H / H A^{+}$is always cosemisimple. This quotient is the third term of the "exact sequence" which completes the inclusion $A \rightarrow H$, and the question of whether or not $C$ is cosemisimple arises naturally in the course of the proof of Theorem 2.1, which shows immediately that the answer is affirmative when $\mathrm{HA}^{+}$happens to be an ideal (both left and right).

Finally, in Section 3 we show that when the ambient Hopf algebra $H$ is CQG, the "expectation" $H \rightarrow A$ that plays a crucial role in the preceding section is positive. In the course of the proof we use a sort of " $A$-relative" Fourier transform from $H$ to $C^{*}$ (whereas ordinary Fourier transforms, as in, say, [Podleś and Woronowicz 1990], are roughly speaking more like maps from $H$ to the dual $H^{*}$ ). This construction has some of the familiar properties from harmonic analysis, such as intertwining products and "convolution products" (Proposition 3.11(1)), playing well with $*$ structures (Proposition 3.11(2)), and satisfying a Plancherel-type condition Remark 3.12. 


\section{Preliminaries}

In this section we make the preparations necessary to prove the main results. Throughout, we work over a fixed field $k$, so all algebras and coalgebras are to be taken over $k$. The reader should feel free to assume $k$ to be algebraically closed whenever convenient, as most results are invariant under scalar extension. In Section 3 we specialize to characteristic zero.

We assume basic familiarity with coalgebra and Hopf algebra theory, for example as presented in [Montgomery 1993]. We will make brief use of the notion of coring over a (not necessarily commutative) $k$-algebra; we refer to [Brzezinski and Wisbauer 2003] for basic properties and results.

The notation is standard: $\Delta_{C}$ and $\varepsilon_{C}$ stand for comultiplication and the counit of the coalgebra $C$ respectively, and we will allow ourselves to drop the subscript when it is clear which coalgebra is being discussed. Similarly, $S_{H}$ or $S$ stands for the antipode of the Hopf algebra $H, 1_{A}$ (or just 1) will be the unit of the algebra $A$, etc. Sweedler notation for comultiplication is used throughout: $\Delta(h)=h_{1} \otimes h_{2}$, as well as for left or right coactions: if $\rho: N \rightarrow N \otimes C(\rho: N \rightarrow C \otimes N)$ is a right (left) $C$-comodule structure, we write $n_{0} \otimes n_{1}\left(n_{\langle-1\rangle} \otimes n_{\langle 0\rangle}\right)$ for $\rho(n)$. We sometimes adorn the indices with parentheses, as in $\Delta(c)=c_{(1)} \otimes c_{(2)}$.

We will also be working extensively with categories of (co)modules over (co)algebras, as well as categories of objects admitting both a module and a comodule structure, compatible in some sense that will be made precise below (see Section 1A). These categories are always denoted by the letter $\mathcal{M}$, with left (right) module structures appearing as left (right) subscripts, and left (right) comodule structures appearing as left (right) superscripts. All such categories are abelian (and in fact Grothendieck), and the forgetful functor from each of them to vector spaces is exact. The one exception from this notational convention is the category of $k$-vector spaces, which we simply call VEC.

Recall that the category $\mu_{f}^{H}$ of finite-dimensional right comodules over a Hopf algebra is monoidal left rigid: every object $V$ has a left dual $V^{*}$ (at the level of vector spaces it is just the usual dual vector space), and one has adjunctions $\left(\otimes V, \otimes V^{*}\right)$ and $\left(V^{*} \otimes, V \otimes\right)$ (the left-hand member of the pair is the left adjoint) on $M_{f}^{H}$.

We also use the correspondence between subcoalgebras of a Hopf algebra $H$ and finite-dimensional (right) comodules over $H$ : for such a comodule $V$, there is a smallest subcoalgebra $D=\operatorname{COALG}(V) \leq H$ such that the structure map $V \rightarrow V \otimes H$ factors through $V \rightarrow V \otimes D$. Conversely, if $D \leq H$ is a simple subcoalgebra, then we denote by $V_{D}$ the simple right $D$-comodule, viewed as a right $H$-comodule. Then, for simple subcoalgebras $D, E \leq H$, the product $E D$ will be precisely $\operatorname{COALG}\left(V_{E} \otimes V_{D}\right)$, while $S(D)$ is $\operatorname{COALG}\left(V^{*}\right)$. 
For a coalgebra $C$, the symbol $\widehat{C}$ denotes the set of isomorphism classes of simple (right, unless specified otherwise) $C$-comodules.

1A. Descent data and adjunctions. We will be dealing with the kind of situation studied extensively in [Takeuchi 1979]: $H$ will be a Hopf algebra, and for most of this section (and in fact the paper), $\iota: A \rightarrow H$ will be a right coideal subalgebra, while $\pi: H \rightarrow C$ will be a quotient left $H$-module coalgebra. Recall that this means that $A$ is a right coideal of $H\left(\Delta_{H}(A) \leq A \otimes H\right)$ as well as a subalgebra, and so the induced map $A \rightarrow A \otimes H$ is an algebra map; similarly, $C$ is the quotient of $H$ by a left ideal as well as a coalgebra, and the induced map $H \otimes C \rightarrow C$ is supposed to be a coalgebra map.

Given a coalgebra map $\pi: H \rightarrow C$, we write $\bar{h}$ for $\pi(h), h \in H$. In this situation, $H$ will naturally be both a left and a right $C$-comodule (via the structure maps $(\pi \otimes \mathrm{id}) \circ \Delta_{H}$ and (id $\left.\otimes \pi\right) \circ \Delta_{H}$ respectively), while $C$ has a distinguished grouplike element $\overline{1}$, where $1 \in H$ is the unit. Write

$$
{ }^{\pi} H={ }^{C} H\left\{h \in H \mid \overline{h_{1}} \otimes h_{2}=\overline{1} \otimes h\right\}, \quad H^{\pi}=H^{C}=\left\{h \in H \mid h_{1} \otimes \overline{h_{2}}=h \otimes \overline{1}\right\} .
$$

These are what were called $\operatorname{LKER}(\pi)$ and $\operatorname{RKER}(\pi)$ back in the introduction, following the notation in [Andruskiewitsch and Devoto 1995]. They are the spaces of $\overline{1}$-coinvariants under the left and right coaction of $C$ on $H$ respectively, in the sense of [Brzezinski and Wisbauer 2003, Section 28.4].

Dually, let $\iota: A \rightarrow H$ be an algebra map, and set $A^{+}=\iota^{-1}\left(\operatorname{ker} \varepsilon_{H}\right)$. Write $H_{\iota}=H_{A}$ for the left $H$-module $H / H \iota\left(A^{+}\right)$, and similarly, ${ }_{\iota} H={ }_{A} H=H / \iota\left(A^{+}\right) H$.

It is now an easy exercise to check that if $\iota: A \rightarrow H$ is a right coideal subalgebra, then $H_{A}$ is a quotient left module coalgebra, and, vice versa, if $\pi: H \rightarrow C$ is the projection on a quotient left module coalgebra, then ${ }^{C} H$ is a right coideal subalgebra of $H$.

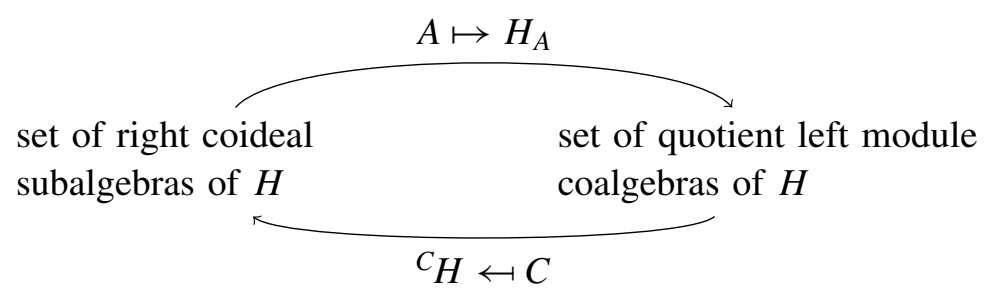

In the above diagram, the maps are order-reversing with respect to the obvious poset structures on the two sets (whose partial orders we write as $\preceq$ )

Remark 1.1. Note that the two order-reversing maps form a Galois connection in the sense of [Mac Lane 1998, Section IV.5] between the poset of right coideal subalgebras and the poset of left module quotient coalgebras. 
Definition 1.2. Let $\iota: A \rightarrow H$ be a right coideal subalgebra and $\pi: H \rightarrow C$ a quotient left module coalgebra. We call $\pi: H \rightarrow H_{A}$ (or $H_{A}$ itself) the right reflection of $\iota: A \rightarrow H$ or of $A$, and $\iota:{ }^{C} H \rightarrow H$ (or ${ }^{C} H$ itself) the left reflection of $\pi: H \rightarrow C$. We also write $r(A)$ and $r(C)$ for $H_{A}$ and ${ }^{C} H$.

Using this language, recall from [Andruskiewitsch and Devoto 1995, Proposition 1.2.3]:

Definition 1.3. Let $H$ be a Hopf algebra. For a right coideal subalgebra $A \rightarrow H$ and a quotient left module coalgebra $H \rightarrow C$, we say that $k \rightarrow A \rightarrow H \rightarrow C \rightarrow k$ is exact if $A$ and $C$ are each other's reflections.

We usually drop the $k$ and talk just about exact sequences $A \rightarrow H \rightarrow C$.

If $H$ is a Hopf algebra and $C$ is a left $H$-module coalgebra, then ${ }_{H}^{C} \mu$ will be the category of left $H$-modules endowed with a left $C$-comodule structure which is a left $H$-module map from $M$ to $C \otimes M$ (where the latter has the left $H$-module structure induced by the comultiplication on $H$ ). Similarly, if $A$ is a right $H$-comodule algebra, then $M_{A}^{H}$ is the category of right $H$-comodules with a right $A$-module structure such that $M \otimes A \rightarrow M$ is a map of right $H$-comodules. The morphisms in each of these categories are required to preserve both structures.

Let $\iota: A \rightarrow H$ be a right coideal subalgebra and $\pi: H \rightarrow C$ a quotient left module coalgebra such that $\pi \circ \iota$ factors through $A \ni a \mapsto \varepsilon(a) \overline{1} \in C$ (this is equivalent to saying that $A \preceq r(C)$, or $C \preceq r(A)$, in the two posets discussed before Definition 1.2). Then, there is an adjunction between the categories ${ }_{A} \mathcal{M}$ and ${ }_{H}^{C} \mathcal{M}$, and dually, an adjunction between $\mu_{A}^{H}$ and $\mu^{C}$. We will recall briefly how these are defined, omitting most of the proofs, which are routine.

Let $M \in{ }_{A} M$. The vector space $H \otimes_{A} M$ then has a left $H$-module structure, as well as a left $C$-comodule structure inherited from the left $C$-coaction on $H$ (checking this is where the condition $A \preceq r(C)$ is needed). This defines a functor $L:{ }_{A} \mathcal{M} \rightarrow{ }_{H}^{C} \mathcal{M}$. To go in the other direction, for $N \in{ }_{H}^{C} \mathcal{M}$, let

$$
R(N)=\left\{n \in N \mid n_{\langle-1\rangle} \otimes n_{\langle 0\rangle}=\overline{1} \otimes n\right\} .
$$

This defines a functor, and, as the notation suggests, $L$ is a left adjoint to $R$.

For the other adjunction, given $M \in M_{A}^{H}$, define $L^{\prime}(M)=M / M A^{+}$. This is a functor (with the obvious definition on morphisms), and it is left adjoint to $R^{\prime}: M^{C} \rightarrow M_{A}^{H}$ defined by $R^{\prime}(N)=N \square_{C} H$; the latter has a right $H$-comodule structure obtained by making $H$ coact on itself, as well as a right $A$-module structure obtained from the right $A$-action on $H$.

Let us now focus on the adjunction ${ }_{A} \mathcal{M} \longleftrightarrow{ }_{H}^{C} \mathcal{M}$. In [Takeuchi 1979], the same discussion is carried out in a slightly less general situation: the adjunction described above is considered in the case $A=r(C)$. On the other hand, we remark that when $C=r(A)$, the category ${ }_{H}^{C} \mathcal{M}$ introduced above is nothing but the category of 
descent data for the ring extension $A \rightarrow H$. Recall from [Brzezinski and Wisbauer 2003, Proposition 25.4] that in our case this would be the category $H_{A} H \mathcal{M}$ of left comodules over the canonical $H$-coring $H \otimes_{A} H$ associated to the algebra extension $A \rightarrow H$. This means left $H$-modules $M$ with an appropriately coassociative and counital left $H$-module map $\rho: M \mapsto\left(H \otimes_{A} H\right) \otimes_{H} M \cong H \otimes_{A} M$.

The usual bijection

$$
H \otimes H \cong H \otimes H, \quad h \otimes k \mapsto h_{1} \otimes h_{2} k
$$

is easily seen to descend to a bijection $H \otimes_{A} H \cong r(A) \otimes H$. Hence, we see that a map $\rho$ as above is the same thing as a map $\psi: M \mapsto r(A) \otimes M$. The other properties of $\rho$, namely, being a coassociative, counital, left $H$-module map, precisely translate to $\psi$ being coassociative, counital, and a left $H$-module map, respectively. Taking into account this equivalence ${ }_{H}^{r(A)} \mathcal{M} \simeq{ }^{H \otimes_{A} H} \mathcal{M}$, the adjunction $(L, R):{ }_{A} \mathcal{M} \longleftrightarrow{ }_{H}^{r(A)} \mathcal{M}$ is an equivalence as soon as $H$ is right faithfully flat over $A$ (this is the faithfully flat descent theorem; see [Nuss 1997, Theorem 3.8]).

Apart from faithful flatness, other criteria are known to ensure $(L, R)$ is an equivalence. To state one such, we recall some notation from [Mesablishvili 2006].

For a ring $A$, consider the contravariant endofunctor ${ }_{A} C_{A}$ on the category of $A$-bimodules defined by

$$
{ }_{A} C_{A}(M)=\operatorname{Hom}(M, \mathbb{Q} / \mathbb{Z}) ;
$$

these are homomorphisms of abelian groups, with the usual $A$-bimodule structure induced from that on $M$. Then, [ibid., Theorem 8.1] (very slightly rephrased) reads:

Theorem. If $\iota: A \rightarrow H$ is a map of rings such that ${ }_{A} C_{A}(\iota):{ }_{A} C_{A}(H) \rightarrow{ }_{A} C_{A}(A)$ is a split epimorphism, then $H \otimes_{A}$ is an equivalence between ${ }_{A} M$ and ${ }^{H \otimes_{A} H} \mathcal{M}$.

Since we have just observed that in our case the functor $H \otimes_{A}$ from the statement of the theorem can be identified with $L:{ }_{A} \mathcal{M} \rightarrow{ }_{H}^{r(A)} \mathcal{M}$, we get the following result as a consequence:

Proposition 1.4. With the previous notation, $(L, R):{ }_{A} \mathcal{M}{ }_{H}^{r(A)} \mathcal{M}$ is an equivalence if the inclusion $\iota: A \rightarrow H$ splits as an A-bimodule map.

Remark 1.5. The paper [Mesablishvili 2006] deals with rings rather than Hopf algebras. To deduce Proposition 1.4 one uses the noted identification ${ }_{H}^{r(A)} \mathcal{M} \simeq{ }^{H \otimes_{A} H} \mathcal{M}$ to turn the problem into the usual formulation of descent for arbitrary rings. Sections 7 and 8 of [ibid.] spell this out.

As a kind of converse to the faithfully flat descent theorem, $(L, R)$ being an equivalence implies that $H$ is right $A$-faithfully flat. Indeed, $H \otimes_{A}$ is then exact on ${ }_{A} \mathcal{M}$. Note that we are using the fact that ${ }_{H}^{r(A)} \mathcal{M}$ is abelian, with the same exact sequences as VEC. All in all, this proves: 
Proposition 1.6. Let $\iota: A \rightarrow H$ be a right coideal subalgebra. The adjunction

$$
(L, R):{ }_{A} \mathcal{M}{ }_{H}^{r(A)} \mathcal{M}
$$

is an equivalence if and only if $H$ is right A-faithfully flat.

Remark 1.7. This result is very similar in spirit to the equivalence (5) $\Longleftrightarrow$ (3) in [Schneider 1990, Theorem I], or to (1) $\Longleftrightarrow(2)$ in [Schauenburg and Schneider 2005, Lemma 1.7]. These can all be deduced from much more general, coringflavored descent theorems that are now available, such as, say, [Caenepeel et al. 2007, Theorem 2.7].

1B. CQG algebras. For background, we rely mainly on [Klimyk and Schmüdgen 1997, 11.3-11.4] or the paper [Dijkhuizen and Koornwinder 1994], where these objects were originally introduced. Recall briefly that these Hopf algebras are meant to have just enough structure to imitate algebras of representative functions on compact groups. This means they are complex $*$-algebras (they possess conjugatelinear involutive multiplication-reversing automorphisms $*$ ) as well as Hopf algebras, and the two structures are compatible in the sense that the comultiplication and the counit are both $*$-algebra homomorphisms.

In addition, CQG algebras are required to have unitarizable comodules. This is a condition we will not spell out in any detail, but it says essentially that every finite-dimensional comodule has an inner product compatible with the coaction in some sense (once more imitating the familiar situation for compact groups, where invariant inner products on representations can be constructed by averaging against the Haar measure). In particular, CQG algebras are automatically cosemisimple, and hence fit comfortably into the setting of Section 2.

Not all $*$-algebras have enveloping $C^{*}$-algebras, but CQG algebras do. See, e.g., [Klimyk and Schmüdgen 1997, Section 11.3.3]. Such a completion is a so-called full, or universal, $C^{*}$-algebraic compact quantum group, in the sense that it is a (unital) $C^{*}$-algebra $A$ endowed with coassociative $C^{*}$-algebra homomorphism $A \rightarrow A \otimes A$ (minimal $C^{*}$ tensor product) with additional conditions ([Klimyk and Schmüdgen 1997, Section 11.3.3, Proposition 32] or [Dijkhuizen and Koornwinder 1994, §4-5]).

On the very few occasions when tensor product $C^{*}$-algebras come up, $\otimes$ always denotes the smallest $C^{*}$ tensor product (as treated in [Wegge-Olsen 1993, T.5], for instance). The term completely positive map between $C^{*}$-algebras will also make brief appearances. Recall that a linear map $T: A \rightarrow B$ between $C^{*}$-algebras is said to be positive if for each $x \in A$ we have $T\left(x^{*} x\right)=y^{*} y$ for some $y \in B$, and completely positive [Takesaki 2002, Section IV.3] if the maps

$$
\mathrm{id} \otimes T: M_{n} \otimes A \rightarrow M_{n} \otimes B
$$

between matrix algebras are all positive. 


\section{Main results}

We now prove the statement from the title of the paper:

Theorem 2.1. A cosemisimple Hopf algebra is faithfully flat over all its Hopf subalgebras.

Proof. Let $H$ be cosemisimple, and $\iota: A \rightarrow H$ an inclusion of a Hopf subalgebra. Combining Propositions 1.6 and 1.4, it suffices to show that $\iota$ splits as an $A$ bimodule map. In fact, one can even find a subcoalgebra $B \leq H$ with $H=A \oplus B$ as $A$-bimodules.

Let $I$ be the set of simple subcoalgebras of $H$, and $J$ the subset of $I$ consisting of subcoalgebras contained in $A$. One then has $H=\bigoplus_{I} D$ and $A=\bigoplus_{J} D$. Define $B=\bigoplus_{I \backslash J} D$; in other words, $B$ is the direct sum of those simple subcoalgebras of $H$ which are not in $A$. Clearly, $B$ is a subcoalgebra and $H=A \oplus B$, and we now only need to check that $B$ is invariant under (either left or right) multiplication by $A$.

Let $D \in J$ and $E \in I \backslash J$ be simple subcoalgebras of $A$ and $B$ respectively. The product $E D$ inside $H$ is then $\operatorname{COALG}\left(V_{E} \otimes V_{D}\right)$ (see last paragraph above Section 1A). Now assume $F \in J$ is a summand of $E D$. This means $V_{F} \leq V_{E} \otimes V_{D}$, so $V_{E}^{*} \leq V_{D} \otimes V_{F}^{*}$. This is absurd: $V_{E}^{*}$ is a $B$-comodule, while $V_{D} \otimes V_{F}^{*}$ is an $A$-comodule.

Remark 2.2. This proves the first part of [Wang 2009, Conjecture 1]; the second part, stating the faithful coflatness of a CQG algebra over quotient CQG algebras, follows immediately from the cosemisimplicity of CQG algebras.

Remark 2.3. Examples of cosemisimple Hopf algebras which are not faithfully coflat over quotient Hopf algebras abound, at least in characteristic zero.

Indeed, let $G$ be a reductive complex algebraic group and $B$ a Borel subgroup.

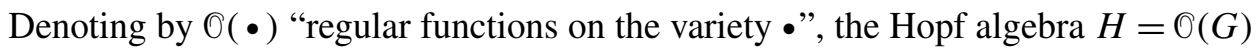
is cosemisimple (e.g., [Fogarty 1969, p. 178]), and it surjects onto $C=\mathscr{O}(B)$.

If the surjection $H \rightarrow C$ were to be faithfully coflat, then, by [Takeuchi 1979, Theorem 2], we could reconstruct $C$ as $H / H A^{+}$for $A=r(C)$. But $A$ is simply the algebra of global regular functions on the projective variety $G / B$, and hence consists only of constants; this provides the contradiction.

In fact, the result can be strengthened slightly. Recall that the coradical $C_{0}$ of a coalgebra $C$ is the sum of all its simple subcoalgebras.

Corollary 2.4. A Hopf algebra $H$ whose coradical $H_{0}$ is a Hopf subalgebra is faithfully flat over its cosemisimple Hopf subalgebras.

Proof. Any cosemisimple Hopf subalgebra $A \leq H$ will automatically be contained in the coradical $H_{0}$. By the previous corollary, $H_{0}$ is faithfully flat over $A$. On the 
other hand, Hopf algebras are faithfully flat (and indeed free) over sub-bialgebras which contain the coradical [Radford 1977b, Corollary 1]; in particular, in this case, $H$ is faithfully flat over $H_{0}$. The conclusion follows.

Now let us place ourselves in the setting of Theorem 2.1, assuming in addition that the Hopf subalgebra $A \rightarrow H$ is conormal in the language of [Andruskiewitsch and Devoto 1995]. This simply means that $\mathrm{HA}^{+}=A^{+} H$, and is equivalent to $C=r(A)$ being a quotient Hopf algebra of $H$ rather than just a quotient coalgebra [Andruskiewitsch and Devoto 1995, Definition 1.1.9]. Recalling the decomposition $H=A \oplus B$ as a direct sum of subcoalgebras, $C$ breaks up as the direct sum of the coalgebras $k=k \overline{1}$ and $B / B A^{+}$. In other words, the coalgebra spanned by the unit of the Hopf algebra $C$ has a coalgebra complement in $C$. It follows from the equivalence of (c) and (f) in [Sweedler 1969, Theorem 14.0.3] that $C$ is a cosemisimple Hopf algebra. Our aim in the rest of this section is to extend this result to the general case covered by Theorem 2.1:

Theorem 2.5. If $\iota: A \rightarrow H$ is a Hopf subalgebra of a cosemisimple Hopf algebra $H$, then the coalgebra $C=r(A)$ is cosemisimple.

Proof. We know from Theorem 2.1 that $H$ is right $A$-faithfully flat, and hence also left faithfully flat (just flip everything by means of the bijective antipode). This then implies, for example by [Takeuchi 1979, Theorem 1], that the second adjunction we introduced above, $\left(L^{\prime}, R^{\prime}\right): M_{A}^{H} \longleftrightarrow M^{C}$, is an equivalence. It is then enough to show that all objects of the category $\mu_{A}^{H}$ are projective, and this is precisely what the next two results do.

Definition 2.6. An object of $\mu_{A}^{H}$ is said to be A-projective if it is projective as an A-module.

Proposition 2.7. Under the hypotheses of Theorem 2.5, every object of $\mu_{A}^{H}$ is A-projective.

Proof. Let $M \in M_{A}^{H}$ be an arbitrary object. Endow $M \otimes H$ with a right $H$-comodule structure by making $H$ coact on itself, and also a right $A$-module structure by the diagonal right action (i.e., $M \otimes H$ is the tensor product in the monoidal category $M_{A}$ ). It is easy to check that these are compatible in the sense that they make $M \otimes H$ into an object of $M_{A}^{H}$, and the map $\rho: m \mapsto m_{\langle 0\rangle} \otimes m_{\langle 1\rangle} \in M \otimes H$ giving $M$ its right $H$ comodule structure is actually a morphism in $\mu_{A}^{H}$. Similarly, id $\otimes \varepsilon_{H}: M \otimes H \rightarrow M$ is a morphism in $\mu_{A}$, and it splits the inclusion $\rho$. It follows that it is enough to show that the object $M \otimes H \in M_{A}^{H}$ described above is $A$-projective.

Theorem 2.1 says that $H$ is $A$-faithfully flat, and it follows from [Masuoka and Wigner 1994, Corollary 2.9] that it is then (left and right) $A$-projective. This means that $M \otimes H$ can be split embedded (in the category $\mu_{A}$ ) into a direct sum of copies 
of $M \otimes A$ with the diagonal right action of $A$. But

$$
M \otimes A \rightarrow M \otimes A, \quad m \otimes a \mapsto m a_{1} \otimes a_{2}
$$

exhibits an isomorphism from $M \otimes A$ with the right $A$-action on the right tensorand to $M \otimes A$ with the diagonal $A$-action (its inverse is $m \otimes a \mapsto m S\left(a_{1}\right) \otimes a_{2}$ ). This means that in $M_{A}, M \otimes H$ is a direct summand of a direct sum of copies of $A$, thus projective.

Proposition 2.8. Under the hypotheses of Theorem 2.5, A-projective objects of $\mu_{A}^{H}$ are projective.

Before going into the proof, we need some preparation, including additional notation to keep track of the several $A$-module or $H$-comodule structures that might exist on the same object.

As in the proof of Theorem 2.1, denote by $I$ and $J \subseteq J$ the sets of simple right comodules over $H$ and $A$, respectively. Recall that these are also in one-to-one correspondence with the simple subcoalgebras of $H$ and $A$, respectively. We will henceforth denote by $\varphi: H \rightarrow A$ the map which is the identity on $A$ and sends every simple subcoalgebra $D \in I \backslash J$ to 0 .

Notice now that $A$ acts on $H$ (as well as on itself) not just by the usual right regular action, but also by the right adjoint action: $h \triangleleft a=S\left(a_{1}\right) h a_{2}(h \in H, a \in A)$. This gives $H$ and $A$ a second structure as objects in $M_{A}^{H}$. When working with this structure rather than the obvious one, we denote these objects by $H_{\mathrm{ad}}$ and $A_{\mathrm{ad}}$.

Lemma 2.9. (a) For any object $M \in \mathcal{M}_{A}^{H}, M \otimes H_{\mathrm{ad}}$ becomes an object of $M_{A}^{H}$ when endowed with the diagonal A-action (where A acts on $M \in \mathcal{M}_{A}^{H}$ and on $H$ by the right adjoint action) and the diagonal $H$-coaction.

(b) Similarly, $M \otimes A_{\text {ad }} \in \mathcal{M}_{A}^{H}$.

(c) id $\otimes \varphi: M \otimes H_{\mathrm{ad}} \rightarrow M \otimes A_{\mathrm{ad}}$ respects the structures from (a) and (b), and hence is a morphism in $M_{A}^{H}$.

Proof. We will only prove (a); (b) is entirely analogous, while (c) follows immediately, since $\varphi$ clearly preserves both the right $H$-coaction and the adjoint $A$-action.

Proving (a) amounts to checking that the diagram

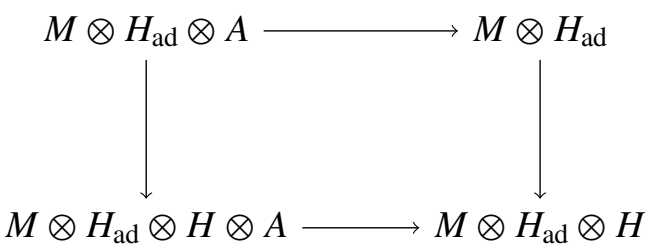


is commutative. The path passing through the upper horizontal line is $m \otimes h \otimes a \longmapsto m a_{1} \otimes S\left(a_{2}\right) h a_{3} \longmapsto m_{0} a_{1} \otimes S\left(a_{4}\right) h_{1} a_{5} \otimes m_{1} a_{2} S\left(a_{3}\right) h_{2} a_{6}$, while the other composition is

$m \otimes h \otimes a \longmapsto m_{0} \otimes h_{1} \otimes m_{1} h_{2} \otimes a \longmapsto m_{0} a_{1} \otimes S\left(a_{2}\right) h_{1} a_{3} \otimes m_{1} h_{2} a_{4}$.

Using the properties of the antipode and counit in a Hopf algebra, we have

$$
\begin{aligned}
m_{0} a_{1} \otimes S\left(a_{4}\right) h_{1} a_{5} \otimes m_{1} a_{2} S\left(a_{3}\right) h_{2} a_{6} & =m_{0} a_{1} \otimes S\left(\varepsilon\left(a_{2}\right) a_{3}\right) h_{1} a_{4} \otimes m_{1} h_{2} a_{5} \\
& =m_{0} a_{1} \otimes S\left(a_{2}\right) h_{1} a_{3} \otimes m_{1} h_{2} a_{4},
\end{aligned}
$$

concluding the proof.

Now denote by $(M \otimes H)^{r} \in M_{A}^{H}$ the object from the proof of Proposition 2.7: the $A$-action is diagonal, while $H$ coacts on the right tensorand alone. The upper $r$ is meant to remind the reader of this.

Lemma 2.10. For $M \in M_{A}^{H}$, the map $\psi_{M}: M \otimes H \rightarrow M \otimes H$ defined by

$$
m \otimes h \longmapsto m_{0} \otimes S\left(m_{1}\right) h
$$

is a morphism in $\mu_{A}^{H}$ from $(M \otimes H)^{r}$ to $M \otimes H_{\mathrm{ad}}$.

Proof. We only check compatibility with the $A$-actions, leaving $H$-coactions to the reader. The composition $(M \otimes H)^{r} \otimes A \longrightarrow(M \otimes H)^{r} \longrightarrow M \otimes H_{\mathrm{ad}}$ is

$$
m \otimes h \otimes a \longmapsto m a_{1} \otimes h a_{2} \stackrel{\psi_{M}}{\longmapsto} m_{0} a_{1} \otimes S\left(m_{1} a_{2}\right) h a_{3},
$$

while the other relevant composition is

$$
m \otimes h \otimes a \stackrel{\psi_{M} \otimes \mathrm{id}}{\longmapsto} m_{0} \otimes S\left(m_{1}\right) h \otimes a \longmapsto m_{0} a_{1} \otimes S\left(a_{2}\right) S\left(m_{1}\right) h a_{3} .
$$

Since $S$ is an algebra antimorphism, they are equal.

Finally, we have:

Lemma 2.11. Let $M \in M_{A}^{H}$. The map $M \otimes A \rightarrow M$ giving $M$ its $A$-module structure is a morphism $M \otimes A_{\mathrm{ad}} \rightarrow M$ in $M_{A}^{H}$.

Proof. Compatibility with the $H$-coactions is built into the definition of the category $M_{A}^{H}$, so one only needs to check that the map is a morphism of $A$-modules. In other words, we must show that the diagram

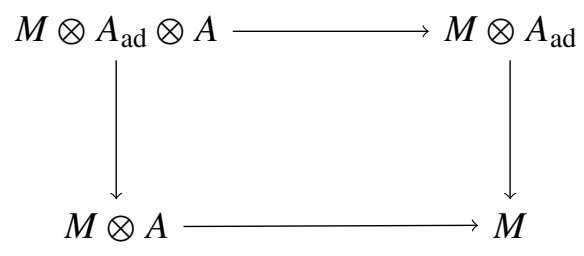


is commutative. The right-down composition is

$$
m \otimes a \otimes b \longmapsto m b_{1} \otimes S\left(b_{2}\right) a b_{3} \longmapsto m b_{1} S\left(b_{2}\right) a b_{3},
$$

while the other composition is

$$
m \otimes a \otimes b \longmapsto m a \otimes b \longmapsto m a b ;
$$

they are thus equal.

Lemma 2.12. For $M \in M_{A}^{H}$, the composition

$$
t_{M}:(M \otimes H)^{r} \stackrel{\psi_{M}}{\longrightarrow} M \otimes H_{\mathrm{ad}} \stackrel{\mathrm{id} \otimes \varphi}{\longrightarrow} M \otimes A_{\mathrm{ad}} \longrightarrow M,
$$

where the last arrow gives $M$ its $A$-module structure, is a natural transformation from the $\mu_{A}^{H}$-endofunctor $(\bullet \otimes H)^{r}$ to the identity functor, and it exhibits the latter as a direct summand of the former.

Proof. The fact that $t_{M}$ is a map in $M_{A}^{H}$ follows from Lemmas 2.9, 2.10 and 2.11. Naturality is immediate (one simply checks that it holds for each of the three maps), as is the fact that $t_{M}$ is a left inverse of the map $M \rightarrow(M \otimes H)^{r}$ giving $M$ its $H$-comodule structure.

We are now ready to prove the result we were after:

Proof of Proposition 2.8. Let $P \in \mathcal{M}_{A}^{H}$ be an $A$-projective object. We must show that $\mathcal{M}_{A}^{H}(P, \bullet)$ is an exact functor. Embedding the identity functor as a direct summand into $(\bullet \otimes H)^{r}$ (Lemma 2.12), it suffices to show that $\mathcal{M}_{A}^{H}\left(P,(\bullet \otimes H)^{r}\right)$ is exact.

The functor $(\bullet \otimes H)^{r}: \mathcal{M}_{A} \rightarrow \mathcal{M}_{A}^{H}$ is right adjoint to forget $: \mathcal{M}_{A}^{H} \rightarrow \mathcal{M}_{A}$ (as $\mu_{A}^{H}$ is the category of coalgebras for the comonad $\bullet \otimes H$ on $\mu_{A}$; see [Mac Lane 1998, Theorem VI.2.1]), so $\mu_{A}^{H}\left(P,(\bullet \otimes H)^{r}\right)$ is naturally isomorphic to $M_{A}(P, \bullet)$, which is exact by our assumption that $P$ is $A$-projective.

Remark 2.13. In the above proof, the forgetful functor forget : $\mu_{A}^{H} \rightarrow \mu_{A}$ has been suppressed in several places, in order to streamline the notation; we trust that this has not caused any confusion.

Remark 2.14. The proof of Proposition 2.7 is essentially a rephrasing of the usual proof that Hopf algebras $H$ with a (right, say) integral sending $1_{H}$ to 1 are cosemisimple [Sweedler 1969, §14.0]; we will call such integrals unital. The map $\varphi: H \rightarrow A$ introduced in Lemma 2.9 might be referred to as an $A$-valued right integral (by which we mean a map preserving both the right $H$-comodule structure and the right adjoint action of $A$ ), and specializes to a unital integral when $A=k$. In conclusion, one way of stating Proposition 2.8 would be:

If the inclusion $\iota: A \rightarrow H$ of a right coideal subalgebra is split by an A-valued right integral, then the forgetful functor $\mu_{A}^{H} \rightarrow M_{A}$ reflects projectives. 
Remark 2.15. Propositions 2.7 and 2.8 can both be traced back to work by Y. Di, but we have included proofs for completeness. Proposition 2.7, for instance, is a consequence of [Doi 1983, Theorem 4]. Similarly, Proposition 2.8 follows from [Doi 1990, Theorem 1]. I thank the referee for pointing this out.

\section{Expectations on CQG subalgebras are positive}

We now move the entire $A \rightarrow H \rightarrow C$ setting over to the case when $H$ is a CQG algebra. We take for granted the preceding sections, and in particular the fact that $C$ is cosemisimple (Theorem 2.5). The inclusion $\iota: A \rightarrow H$ is now one of *-algebras, and we follow the operator-algebraists' convention of referring to its left inverse $p: H \rightarrow A$ from the proof of Theorem 2.1 as the expectation of $H$ on $A$ (in accordance with a view of $A$ and $H$ as consisting of random variables on noncommutative measure spaces). Positivity here means the following:

Think of $H$ as embedded in its universal $C^{*}$ completion $H_{u}$ (Section 1B), and complete $A$ to $A_{u}$ with the subspace norm. Then, $p$ extends to a completely positive map $H_{u} \rightarrow A_{u}$. Equivalently, the self-map $\iota \circ p: H \rightarrow H$ lifts to a completely positive self-map of $H_{u}$.

Note that a functional $\psi \in H^{*}$ with $\psi(1)=1$ extends to a state on the $C^{*}$ completion $H_{u}$ if and only if it is positive in the usual sense; i.e., $\psi\left(x^{*} x\right) \geq 0$ for every $x \in H$.

The main result of the section is this:

Theorem 3.1. Let $\iota: A \rightarrow H$ be an inclusion of $C Q G$ algebras. Then, the expectation $p: H \rightarrow A$ is positive in the above sense.

Remark 3.2. So-called expected $C^{*}$-subalgebras of (locally) compact quantum groups have featured prominently in the literature (see [Tomatsu 2007; Salmi and Skalski 2012] and references therein). The techniques used in the proof of Theorem 3.1 will be applied elsewhere to characterize all right coideal $*$-subalgebras $A$ of a CQG algebra $H$ which are expected in the sense of admitting a positive splitting of the inclusion as an $A$-bimodule, right $H$-comodule map, where positivity is understood as in Theorem 3.1.

Let us first reformulate the theorem slightly. Denote the unique unital (left and right) integral of $C$ by $h_{C}$, and the composition $h_{C} \circ \pi$ by $\varphi$ (where $\pi: H \rightarrow C$ is the surjection we start out with). The expectation decomposes as $(\varphi \otimes \mathrm{id}) \circ \Delta$ : $H \rightarrow A$. This follows easily from the decomposition $H=A \oplus B$ as a direct sum of subcoalgebras used in the proof of Theorem 2.1, and the fact that $\left.\varphi\right|_{A}$ equals $\varepsilon_{A}$ and $\left.\varphi\right|_{B}$ is the zero map.

Remark 3.3. Let us note in passing that $\varphi$ is self-adjoint as a functional, in the sense that $\varphi\left(x^{*}\right)$ is the complex conjugate of $\varphi(x)$ for any $x \in H$. This follows 
immediately from $\left.\varphi\right|_{A}=\varepsilon_{A}$ and $\left.\varphi\right|_{B}=0$, the fact that $A$ and $B$ are closed under $*$, and the fact that $\varepsilon$ is a $*$-homomorphism.

This observation is needed in the proof of item (2) in Proposition 3.11, for instance.

Lemma 3.4. The conclusion of Theorem 3.1 holds if and only if the functional $\varphi \in H^{*}$ is positive.

Proof. Note that $\varphi$ equals $\varepsilon \circ p$ (more pedantically, in this expression $\varepsilon$ is the restriction of $\varepsilon_{H}$ to $A$ ). If $p$ is positive then so is $\varphi$, given that $\varepsilon$ is a $*$-algebra map $A \rightarrow \mathbb{C}$ which lifts to $A_{u}$.

Conversely, if $\varphi$ is positive (and hence lifts to a state on $H_{u}$ ), then both maps in the composition $(\varphi \otimes \mathrm{id}) \circ \Delta: H \rightarrow H$ lift to completely positive maps on the appropriate $C^{*}$ completions ( $\Delta$ lifts to a $C^{*}$-algebra map $H_{u} \rightarrow H_{u} \otimes H_{u}$, while $\varphi \otimes$ id : $H_{u} \otimes H_{u} \rightarrow H_{u}$ will also be completely positive). But that composition is precisely $\iota \circ p$, as noted above.

Remark 3.5. The identity $\varphi=\varepsilon \circ p$, in particular, shows that $\varphi \circ S=\varphi$. This is needed below.

We are going to take what looks like a detour to make the necessary preparations.

For a cosemisimple coalgebra $D$ over an algebraically closed field, denote by $D^{\bullet}$ its restricted dual: the direct sum of the matrix algebras dual to the matrix subcoalgebras of $D$. In general, $D^{\bullet}$ is a nonunital algebra. In our case, the full dual $H^{*}$ is in addition a (unital) $*$-algebra, with $*$ operation defined by

$$
f^{*}(x)=f\left((S x)^{*}\right)^{*} \text { for all } x \in H,
$$

where the outer $*$ means complex conjugation of a number (see, e.g., [Van Daele 1998, Proposition 4.3]). Furthermore, $C^{\bullet} \leq H^{*}$ is a $*$-subalgebra.

Finally, again for a cosemisimple coalgebra $D$, we will talk about its completion $\bar{D}$; this is by definition the direct product of the matrix subcoalgebras comprising $D$. Equivalently, $\bar{D}$ is the (ordinary, vector space) dual of $D^{\bullet}$. The module structure $H \otimes C \rightarrow C$ extends to an action of $H$ on $\bar{C}$.

Remark 3.6. This extension of the $H$-module structure to $\bar{C}$ is a simple enough observation, but there is some content to it. The claim is that for $x \in H$ and some simple subcoalgebra $C_{\alpha} \leq C$ (for $\alpha \in \widehat{C}$ ), there are only finitely many simple comodules $\beta \in \widehat{C}$ such that $x C_{\beta}$ intersects $C_{\alpha}$ nontrivially.

Although $M^{C}$ is not monoidal, $V \otimes W$ can be made sense of as a $C$-comodule for any $H$-comodule $V$ and $C$-comodule $W$. This makes $\mu^{C}$ into a module category over the monoidal category $\mu^{H}$. Upon rephrasing the claim using the correspondence $W \mapsto \operatorname{COALG}(W)$ between comodules and subcoalgebras, it reads: for each finite-dimensional $H$-comodule $V$ and each $\alpha \in \widehat{C}$, there are only finitely many $\beta \in \widehat{C}$ 
such that (identifying $\alpha, \beta$ with the corresponding comodules) $\operatorname{Hom}_{\mathcal{M}^{c}}(\alpha, V \otimes \beta)$ is nonzero. But just as in a rigid monoidal category, $V \otimes: \mu^{C} \rightarrow \mu^{C}$ is right adjoint to $V^{*} \otimes$, and hence we're saying only finitely many $\beta$ satisfy $\operatorname{Hom}\left(V^{*} \otimes \alpha, \beta\right) \neq 0$. This is clear simply because $V^{*} \otimes \alpha$ is some finite direct sum of irreducibles.

First, a preliminary result:

Lemma 3.7. The squared antipode $S^{2}$ of $H$ descends to an automorphism of every simple subcoalgebra $C_{\alpha}$ of $C$. Moreover, the resulting automorphism on the $C^{*-}$ algebra $C_{\alpha}^{*}$ is conjugation by an invertible positive operator.

Proof. That $S^{2}$ descends to $C=H / H A^{+}$is clear from the fact that it acts on $A$. We move the action over to duals by precomposition: $S^{2} f=f\left(S^{2} \cdot\right)$ for $f \in H^{*}$.

Now let $D \leq H$ be a simple subcoalgebra, and $\bigoplus_{\alpha \in I} C_{\alpha}, I \subset \widehat{C}$ the image of $D$ through $H \rightarrow C$. The squared antipode acts on $D^{*}$ as conjugation by a positive operator $F$ [Klimyk and Schmüdgen 1997, Chapter 11, Lemma 30 and Proposition 34], and, by the previous paragraph, preserves the subalgebra $B=\bigoplus_{\alpha \in I} C_{\alpha}^{*}$. In particular, conjugation by $F$ permutes the $|I|$ minimal nonzero projections $p_{\alpha}$, $\alpha \in I$ in the center of $B$. I claim that this permutation action is in fact trivial, which would finish the proof.

To check the claim, consider the unique (up to isomorphism) simple $*$-representation of $D^{*}$ on a Hilbert space $\mathscr{H}$. If $F p_{\alpha} F^{-1}$ were equal to some $p_{\beta}$ with $\beta \neq \alpha \in I$, then $F$ would map the range of $p_{\alpha}$ onto the range of $p_{\beta}$. Denoting by $\langle$,$\rangle the$ inner product on $\mathscr{H}$, this implies that $\langle F x, x\rangle$ vanishes for any $x$ in the range of $p_{\alpha}$. This cannot happen for nonzero $x$, as $F$ is both positive and invertible.

We now establish the existence of a kind of "relative Haar measure" on $C^{\bullet}$.

Proposition 3.8. There is an element $\theta \in \bar{C}$ satisfying the following conditions:

(a) Writing $\theta$ as a formal sum of elements in the simple subcoalgebras of $C$, its component in $\mathbb{C} \overline{1} \leq C$ is $\overline{1}$.

(b) It is $H$-invariant, in the sense that $x \theta=\varepsilon(x) \theta$ for $x \in H$.

(c) It is positive as a functional on the $*$-algebra $C^{\bullet}$.

Sketch of proof. Let $e_{i}, e^{i}, i \in I$ be dual bases in $C$ and $C^{\bullet}$ respectively, compatible with the decomposition of $C$ into simple subcoalgebras. We distinguish an element $0 \in I$ such that $e_{0}=\overline{1}$. Since the automorphism $S^{2}$ of $H$ descends to $C=H / H A^{+}$, the definition

$$
\theta=\sum_{i \in I} e^{i}\left(S^{2} e_{i(2)}\right) e_{i(1)}
$$

makes sense as an element of $\bar{C}$, and clearly satisfies (a). Moreover, the definition does not depend on the choice of bases. 
The calculation proving $H$-invariance can simply be lifted, e.g., from [Van Daele 1997, Proposition 1.1]. Even though that result is about finite-dimensional Hopf algebras, it works verbatim in the present setting.

Finally, let us prove positivity, this time imitating [Van Daele 1996]. Let $\alpha \in \widehat{C}$, and $u \in C_{\alpha}^{*} \leq C^{\bullet}$ an element. We can assume harmlessly that the bases $e_{i}, e^{i}$ are organized as matrix (co)units; i.e., those $e_{i}$ in the matrix coalgebra $C_{\alpha}$ form a matrix counit $e_{p q}$, and $e^{i}$ will then be the dual matrix unit $e^{p q} \in C_{\alpha}^{*}$.

Now note that $e_{p q}$, regarded as a functional on $C_{\alpha}^{*}$, can be written as $\operatorname{tr}_{\alpha}\left(\cdot e^{q p}\right)$, where $\operatorname{tr}_{\alpha}$ is the trace on the matrix algebra $C_{\alpha}^{*} \cong M_{n}$, so that $\operatorname{tr}_{\alpha}(1)=n$. In conclusion, the component of $\theta$ in $C_{\alpha}$, regarded as a functional on $C_{\alpha}^{*}$, is

$$
\theta_{\alpha}=\sum_{p, q} \operatorname{tr}_{\alpha}\left(\cdot S^{2}\left(e^{p q}\right) e^{q p}\right)
$$

If $Q \in C_{\alpha}^{*}$ is a positive operator such that conjugation by $Q$ equals $S^{2}$ on $C_{\alpha}^{*}$ (Lemma 3.7), then, suppressing summation over $p, q=1, \ldots, n$,

$$
S^{2}\left(e^{p q}\right) e^{q p}=Q e^{p q} Q^{-1} e^{q p}=\operatorname{tr}_{\alpha}\left(Q^{-1}\right) Q .
$$

This is a positive operator, and the conclusion follows.

Remark 3.9. The expression (3), the invariance of $\theta$ with respect to bases, and the fact that $S^{2}\left(e^{p q}\right)$ are again matrix units make it clear that $\theta \circ S^{2}=\theta$. In fact $\theta$ is unique, but we do not need this stronger fact.

Definition 3.10. Keeping the previous notation, the $\varphi$-relative Fourier transform $\mathscr{F}: H \rightarrow C^{\bullet}$ is defined as

$$
H \ni x \mapsto \varphi(S x \cdot) .
$$

There is a slight abuse of notation in the definition: although a priori $\varphi$ is a functional on $H$, it descends to one on $C=H / H A^{+}$. The map $\mathscr{F}$ is a relative analogue to the usual Fourier transform [Podleś and Woronowicz 1990, §2], and enjoys similar properties. Let us record some of them:

Proposition 3.11. The map $\mathscr{F}: H \rightarrow C^{\bullet}$ introduced above satisfies the following relations:

(1) $\mathscr{F}(x \triangleleft \mathscr{F} y)=\mathscr{F} x \mathscr{F} y$ for all $x, y \in H$, where the right action $\triangleleft$ of $H^{*}$ on $H$ is defined by $x \triangleleft f=f\left(x_{1}\right) x_{2}$.

(2) $\mathscr{F}(x)^{*}=S^{2} \mathscr{F}\left((S x)^{*}\right)$, where the $*$ structure on $C^{\bullet}$ is defined in (2), and $S^{2}$ acts on $H^{*}$ by precomposition, as in the proof of Lemma 3.7.

(3) $\varepsilon(x \triangleleft \mathscr{F} y)=\varphi(\operatorname{Sy} x)$.

(4) $\theta \mathscr{F}=\varepsilon$, where $\theta$ is the functional on $C^{\bullet}$ from Proposition 3.8.

(5) $\mathscr{F} S^{2}=S^{-2} \mathscr{F}$. 
Proof. Most of this consists of simple computations, so let us only prove the first and fourth items.

Applying both sides of (1) to $z \in H$, we have to prove

$$
\varphi\left(S y x_{1}\right) \varphi\left(S x_{2} z\right)=\varphi\left(S x z_{1}\right) \varphi\left(S y z_{2}\right) .
$$

Substituting $y$ for $S y, z$ for $S z$, and using $\varphi \circ S=\varphi$ (Remark 3.5), this turns into

$$
\varphi\left(y x_{1}\right) \varphi\left(z x_{2}\right)=\varphi\left(z_{2} x\right) \varphi\left(y S z_{1}\right) .
$$

Now make the substitution $y x_{1} \otimes x_{2}=a \otimes b$, which in turn means $y \otimes x=a S b_{1} \otimes b_{2}$. The target identity turns into

$$
\varphi(a) \varphi(z b)=\varphi\left(a S b_{1} S z_{1}\right) \varphi\left(z_{2} b_{2}\right) .
$$

Writing $z b=c$, it transforms further into

$$
\varphi(a) \varphi(c)=\varphi\left(a S c_{1}\right) \varphi\left(c_{2}\right) .
$$

Finally, the substitution of $c$ for $S^{-1} c$ and again $\varphi \circ S=\varphi$ turn this into

$$
\varphi(a) \varphi(c)=\varphi\left(a c_{2}\right) \varphi\left(c_{1}\right) .
$$

To prove this last equality, it suffices to split into two cases, according to whether $c$ is in $A$ or the complementary $A$-bimodule, right $H$-comodule $\operatorname{ker}(p)$.

In the latter case, both $\varphi(c)$ and $\varphi\left(c_{1}\right)$ vanish. In the former, the left-hand side is $\varphi(a) \varepsilon(c)$, while the right-hand side is $\varphi(a c)$ (since $\varphi\left(c_{1}\right)=\varepsilon\left(c_{1}\right)$ ). These two expressions are equal because $\varphi=\varepsilon p$ and $p$ is an $A$-bimodule map.

We now check (4). Applying its left-hand side to $x \in H$, we get $\theta(\varphi(S x \cdot))=$ $\varphi(\operatorname{Sx} \theta)$, where this time $\theta$ is thought of as an element of $\bar{C}, S x \theta$ is the action of $S x$ on it (Remark 3.6), and $\varphi$ is regarded naturally as a functional on $\bar{C}$. By the $H$-invariance of $\theta$ (Proposition 3.8(b)), the expression is $\varepsilon(x) \varphi(\theta)=\varepsilon(x)$ by Proposition 3.8(a).

All of the ingredients are now in place.

Proof of Theorem 3.1. According to Lemma 3.4, it suffices to show that $\varphi\left(x^{*} x\right) \geq 0$ for all $x \in H$. We do this through a string of equalities based on the preliminary results of this section.

Let $x, y \in H$. Then, we have

$$
\begin{aligned}
\theta\left((\mathscr{F} y)^{*} \mathscr{F} x\right) & \stackrel{(2)}{=} \theta\left(S^{2} \mathscr{F}\left((S y)^{*}\right) \mathscr{F} x\right)=\theta\left(S^{2} \mathscr{F}\left((S y)^{*}\right) \mathscr{F}\left(S^{2} x\right)\right) \\
& \stackrel{(1)}{=} \theta \mathscr{F}\left((S y)^{*} \triangleleft \mathscr{F}\left(S^{2} x\right)\right) \stackrel{(4)}{=} \varepsilon\left((S y)^{*} \triangleleft \mathscr{F}\left(S^{2} x\right)\right) \\
& \stackrel{(3)}{=} \varphi\left(S^{3} x(S y)^{*}\right)=\varphi\left(S^{3} x S\left(S^{2} y\right)^{*}\right)=\varphi\left(\left(S^{2} y\right)^{*} S^{2} x\right),
\end{aligned}
$$


where the numbers above the equal signs refer to the items in Proposition 3.11, the second equality follows from (5) and the fact that $\theta S^{2}=\theta$ (Remark 3.9), the next-to-last one is a simple manipulation valid in any Hopf $*$-algebra, and the last equality is based on $\varphi S=\varphi$ (Remark 3.5). Since the left-hand side is nonnegative when $x=y$, so is the right-hand side. This concludes the proof of the theorem.

Remark 3.12. The equality obtained in the course of the proof should be thought of as a Plancherel theorem [Rudin 1991, 7.9, p. 188], to the effect that the relative Fourier transform is an isometry with respect to the "inner products" induced by $\varphi$ and $\theta$.

\section{Acknowledgements}

I would like to thank Akira Masuoka and Issan Patri for useful references, conversations and comments on the contents of the paper. I am also grateful to the anonymous referee for bringing to my attention the references [Doi 1983; 1990], and for numerous other suggestions that have helped considerably with improving the manuscript.

\section{References}

[Andruskiewitsch and Devoto 1995] N. Andruskiewitsch and J. Devoto, "Extensions of Hopf algebras", Algebra i Analiz 7:1 (1995), 22-61. MR 96f:16044 Zbl 0857.16032

[Arkhipov and Gaitsgory 2003] S. Arkhipov and D. Gaitsgory, "Another realization of the category of modules over the small quantum group", Adv. Math. 173:1 (2003), 114-143. MR 2004e:17010 Zbl 1025.17004

[Brzezinski and Wisbauer 2003] T. Brzezinski and R. Wisbauer, Corings and comodules, London Mathematical Society Lecture Note Series 309, Cambridge University Press, 2003. MR 2004k:16093 Zbl 1035.16030

[Caenepeel et al. 2007] S. Caenepeel, E. De Groot, and J. Vercruysse, "Galois theory for comatrix corings: descent theory, Morita theory, Frobenius and separability properties", Trans. Amer. Math. Soc. 359:1 (2007), 185-226. MR 2007e:16042 Zbl 1115.16017

[Chirvasitu 2010] A. Chirvasitu, "On epimorphisms and monomorphisms of Hopf algebras", $J$. Algebra 323:5 (2010), 1593-1606. MR 2011f:16075 Zbl 1198.16030

[Demazure and Gabriel 1970] M. Demazure and P. Gabriel, Groupes algébriques, I: Géométrie algébrique, généralités, groupes commutatifs, Masson, Paris, 1970. MR 46 \#1800 Zbl 0203.23401

[Dijkhuizen and Koornwinder 1994] M. S. Dijkhuizen and T. H. Koornwinder, "CQG algebras: a direct algebraic approach to compact quantum groups", Lett. Math. Phys. 32:4 (1994), 315-330. MR 95m:16029 Zbl 0861.17005

[Doi 1983] Y. Doi, “On the structure of relative Hopf modules”, Comm. Algebra 11:3 (1983), 243-255. MR 84a:16014 Zbl 0502.16009

[Doi 1990] Y. Doi, "Hopf extensions of algebras and Maschke type theorems", Israel J. Math. 72:1-2 (1990), 99-108. MR 92b:16078 Zbl 0731.16025

[Fogarty 1969] J. Fogarty, Invariant theory, W. A. Benjamin, New York, 1969. MR 39 \#1458 Zbl 0191.51701 
[Kaplansky 1975] I. Kaplansky, Bialgebras, University of Chicago, 1975. MR 55 \#8087

[Klimyk and Schmüdgen 1997] A. Klimyk and K. Schmüdgen, Quantum groups and their representations, Springer, Berlin, 1997. MR 99f:17017 Zbl 0891.17010

[Mac Lane 1998] S. Mac Lane, Categories for the working mathematician, 2nd ed., Graduate Texts in Mathematics 5, Springer, New York, 1998. MR 2001j:18001 Zbl 0906.18001

[Masuoka and Wigner 1994] A. Masuoka and D. Wigner, "Faithful flatness of Hopf algebras", $J$. Algebra 170:1 (1994), 156-164. MR 95i:16040 Zbl 0820.16034

[Mesablishvili 2006] B. Mesablishvili, "Monads of effective descent type and comonadicity", Theory Appl. Categ. 16:1 (2006), 1-45. MR 2006m:18002 Zbl 1085.18003

[Montgomery 1993] S. Montgomery, Hopf algebras and their actions on rings, CBMS Regional Conference Series in Mathematics 82, American Mathematical Society, Providence, RI, 1993. MR 94i:16019 Zbl 0793.16029

[Nuss 1997] P. Nuss, "Noncommutative descent and non-abelian cohomology", K-Theory 12:1 (1997), 23-74. MR 99d:16001 Zbl 0884.18015

[Oberst and Schneider 1974] U. Oberst and H.-J. Schneider, "Untergruppen formeller Gruppen von endlichem Index", J. Algebra 31 (1974), 10-44. MR 50 \#13057 Zbl 0304.14028

[Podleś and Woronowicz 1990] P. Podleś and S. L. Woronowicz, "Quantum deformation of Lorentz group”, Comm. Math. Phys. 130:2 (1990), 381-431. MR 91f:46100 Zbl 0703.22018

[Radford 1977a] D. E. Radford, “Operators on Hopf algebras”, Amer. J. Math. 99:1 (1977), 139-158. MR 55 \#10505 Zbl 0369.16011

[Radford 1977b] D. E. Radford, "Pointed Hopf algebras are free over Hopf subalgebras", J. Algebra 45:2 (1977), 266-273. MR 55 \#10506 Zbl 0354.16004

[Rudin 1991] W. Rudin, Functional analysis, 2nd ed., McGraw-Hill, New York, 1991. MR 92k:46001 Zbl 0867.46001

[Salmi and Skalski 2012] P. Salmi and A. Skalski, "Idempotent states on locally compact quantum groups", Q. J. Math. 63:4 (2012), 1009-1032. MR 2999995 Zbl 1263.46055

[Schauenburg 2000] P. Schauenburg, "Faithful flatness over Hopf subalgebras: counterexamples", pp. 331-344 in Interactions between ring theory and representations of algebras (Murcia, 1998), edited by F. Van Oystaeyen and M. Saorin, Lecture Notes in Pure and Appl. Math. 210, Dekker, New York, 2000. MR 2001d:16061 Zbl 0977.16019

[Schauenburg and Schneider 2005] P. Schauenburg and H.-J. Schneider, "On generalized Hopf Galois extensions”, J. Pure Appl. Algebra 202:1-3 (2005), 168-194. MR 2006e:16069 Zbl 1081.16045

[Schneider 1990] H.-J. Schneider, "Principal homogeneous spaces for arbitrary Hopf algebras", Israel J. Math. 72:1-2 (1990), 167-195. MR 92a:16047 Zbl 0731.16027

[Sweedler 1969] M. E. Sweedler, Hopf algebras, W. A. Benjamin, New York, 1969. MR 40 \#5705 Zbl 0194.32901

[Takesaki 2002] M. Takesaki, Theory of operator algebras, I, Encyclopaedia of Mathematical Sciences 124, Springer, Berlin, 2002. MR 2002m:46083 Zbl 0990.46034

[Takeuchi 1972] M. Takeuchi, "A correspondence between Hopf ideals and sub-Hopf algebras", Manuscripta Math. 7 (1972), 251-270. MR 48 \#328 Zbl 0238.16011

[Takeuchi 1979] M. Takeuchi, "Relative Hopf modules: equivalences and freeness criteria", J. Algebra 60:2 (1979), 452-471. MR 82m:16006 Zbl 0492.16013

[Tomatsu 2007] R. Tomatsu, "A characterization of right coideals of quotient type and its application to classification of Poisson boundaries", Comm. Math. Phys. 275:1 (2007), 271-296. MR 2008j:46058 Zbl 1130.46042 
[Van Daele 1996] A. Van Daele, "Discrete quantum groups", J. Algebra 180:2 (1996), 431-444. MR 97a:16076 Zbl 0864.17012

[Van Daele 1997] A. Van Daele, "The Haar measure on finite quantum groups", Proc. Amer. Math. Soc. 125:12 (1997), 3489-3500. MR 98b:16036 Zbl 0888.16023

[Van Daele 1998] A. Van Daele, "An algebraic framework for group duality", Adv. Math. 140:2 (1998), 323-366. MR 2000g:16045 Zbl 0933.16043

[Wang 2009] S. Wang, "Simple compact quantum groups, I", J. Funct. Anal. 256:10 (2009), 33133341. MR 2011c:46150 Zbl 1176.46063

[Wegge-Olsen 1993] N. E. Wegge-Olsen, $K$-theory and $C^{*}$-algebras: a friendly approach, Clarendon, New York, 1993. MR 95c:46116 Zbl 0780.46038

[Woronowicz 1987] S. L. Woronowicz, "Compact matrix pseudogroups", Comm. Math. Phys. 111:4 (1987), 613-665. MR 88m:46079 Zbl 0627.58034

[Woronowicz 1988] S. L. Woronowicz, "Tannaka-Krein duality for compact matrix pseudogroups. Twisted SU (N) groups”, Invent. Math. 93:1 (1988), 35-76. MR 90e:22033 Zbl 0664.58044

Communicated by Susan Montgomery

Received 2013-08-11 Revised 2014-03-06 Accepted 2014-04-21

chirvasitua@gmail.com Department of Mathematics, University of Washington, Box 354350, Seattle, WA 98195-4350, United States 


\section{Algebra \& Number Theory}

msp.org/ant

\section{EDITORS}

MANAGING EDITOR

Bjorn Poonen

Massachusetts Institute of Technology

Cambridge, USA

\author{
EDITORIAL BOARD CHAIR \\ David Eisenbud \\ University of California \\ Berkeley, USA
}

BOARD OF EDITORS

Georgia Benkart

Dave Benson

Richard E. Borcherds

John H. Coates

J-L. Colliot-Thélène

Brian D. Conrad

Hélène Esnault

Hubert Flenner

Edward Frenkel

Andrew Granville

Joseph Gubeladze

Roger Heath-Brown

Craig Huneke

Yujiro Kawamata

János Kollár

Yuri Manin

Barry Mazur

Philippe Michel

Susan Montgomery
University of Wisconsin, Madison, USA

University of Aberdeen, Scotland

University of California, Berkeley, USA

University of Cambridge, UK

CNRS, Université Paris-Sud, France

University of Michigan, USA

Freie Universität Berlin, Germany

Ruhr-Universität, Germany

University of California, Berkeley, USA

Université de Montréal, Canada

San Francisco State University, USA

Oxford University, UK

University of Virginia, USA

University of Tokyo, Japan

Princeton University, USA

Northwestern University, USA

Harvard University, USA

École Polytechnique Fédérale de Lausanne

University of Southern California, USA
Shigefumi Mori

Raman Parimala

Jonathan Pila

Anand Pillay

Victor Reiner

Peter Sarnak

Joseph H. Silverman

Michael Singer

Vasudevan Srinivas

J. Toby Stafford

Bernd Sturmfels

Richard Taylor

Ravi Vakil

Michel van den Bergh

Marie-France Vignéras

Kei-Ichi Watanabe

Efim Zelmanov

Shou-Wu Zhang
RIMS, Kyoto University, Japan

Emory University, USA

University of Oxford, UK

University of Notre Dame, USA

University of Minnesota, USA

Princeton University, USA

Brown University, USA

North Carolina State University, USA

Tata Inst. of Fund. Research, India

University of Michigan, USA

University of California, Berkeley, USA

Harvard University, USA

Stanford University, USA

Hasselt University, Belgium

Université Paris VII, France

Nihon University, Japan

University of California, San Diego, USA

Princeton University, USA

\section{PRODUCTION}

production@msp.org

Silvio Levy, Scientific Editor

See inside back cover or msp.org/ant for submission instructions.

The subscription price for 2014 is US $\$ 225 /$ year for the electronic version, and $\$ 400 /$ year ( $\$ 55$, if shipping outside the US) for print and electronic. Subscriptions, requests for back issues and changes of subscribers address should be sent to MSP.

Algebra \& Number Theory (ISSN 1944-7833 electronic, 1937-0652 printed) at Mathematical Sciences Publishers, 798 Evans Hall \#3840, c/o University of California, Berkeley, CA 94720-3840 is published continuously online. Periodical rate postage paid at Berkeley, CA 94704, and additional mailing offices.

ANT peer review and production are managed by EditFLOw ${ }^{\circledR}$ from Mathematical Sciences Publishers.

\section{PUBLISHED BY}

mathematical sciences publishers

nonprofit scientific publishing

http://msp.org/

(C) 2014 Mathematical Sciences Publishers 


\section{Algebra \& Number Theory}

Volume $8 \quad$ No. $5 \quad 2014$

Polarization estimates for abelian varieties

DAVID MASSER and GISBERT WÜSTHOLZ

Compatibility between Satake and Bernstein isomorphisms in characteristic $p$

1071

RACHEL OLLIVIER

The final $\log$ canonical model of $\bar{M}_{6}$

1113

FABIAN MÜLLER

Poisson structures and star products on quasimodular forms

1127

FRANÇOIS DUMAS and EMMANUEL ROYER

Affinity of Cherednik algebras on projective space

GWYN BELLAMY and MAURIZIO MARTINO

ALEXANDRU CHIRVASITU

Tetrahedral elliptic curves and the local-global principle for isogenies

1201

BARINDER S. BANWAIT and JOHN E. CREMONA

Local cohomology with support in generic determinantal ideals

1231

Claudiu RAICU and JERZY WEYMAN

Affine congruences and rational points on a certain cubic surface

1259

PIERRE LE BoudeC 